\title{
Mortality rate among critically ill patients with COVID-19 in a medical system with adequate hospital resources: a prospective observational study
}

\section{Christina Routsi}

Evangelismos Private Hospital

\section{Eleni Magira}

Evangelismos Private Hospital

\section{Stelios Kokkoris}

Geniko Nosokomeio Evangelismou

Ilias Siembos

Geniko Nosokomeio Evangelismou

Charikleia Vrettou

Geniko Nosokomeio Evangelismou

Dimitris Zervakis

Geniko Nosokomeio Evangelismou

Eleni Ischaki

Geniko Nosokomeio Evangelismou

\section{Sotiris Malahias}

Geniko Nosokomeio Evangelismou

loanna Sigala

Geniko Nosokomeio Evangelismou

\section{Andreas Asimakos}

Geniko Nosokomeio Evangelismou

Theodora Daidou

Geniko Nosokomeio Evangelismou

Panagiotis Kaltsas

Geniko Nosokomeio Evangelismou

Evangelia Douka

Geniko Nosokomeio Evangelismou

Adamandia Sotiriou

Geniko Nosokomeio Evangelismou

Vassiliki Markaki

Geniko Nosokomeio Evangelismou 
Prodromos Temberikidis

Geniko Nosokomeio Evangelismou

Apostolos Koroneos

Geniko Nosokomeio Evangelismou

Panagiotis Politis

Geniko Nosokomeio Evangelismou

\section{Zafiria Mastora}

Geniko Nosokomeio Evangelismou

Efrosini Dima

Geniko Nosokomeio Evangelismou

Theodoros Tsoutsouras

Geniko Nosokomeio Evangelismou

loannis Papahatzakis

Geniko Nosokomeio Evangelismou

\section{Panagiota Gioni}

Geniko Nosokomeio Evangelismou

\section{Athina Strilakou}

Geniko Nosokomeio Evangelismou

Aikaterini Maraguti

Geniko Nosokomeio Evangelismou

\section{Eleftheria Mizi}

Geniko Nosokomeio Evangelismou

\section{Ageliki Kanavou}

Geniko Nosokomeio Evangelismou

\section{Aikaterini Sarri}

Geniko Nosokomeio Evangelismou

\section{Evdokia Gavrielatou}

Geniko Nosokomeio Evangelismou

Spyros Mentzelopoulos

Geniko Nosokomeio Evangelismou

loannis Kalomenidis

Geniko Nosokomeio Evangelismou

\section{Vassilios Papastamopoulos}

Geniko Nosokomeio Evangelismou

\section{Anastasia Kotanidou}

Geniko Nosokomeio Evangelismou

Spyros G Zakynthinos ( $D$ szakynthinos@yahoo.com )

National and Kapodistrian University of Athens Faculty of Medicine 
Research

Keywords: Coronavirus, Coronavirus disease 2019, COVID-19, Critical care, Acute hypoxemic respiratory failure, Mortality, Acute respiratory distress syndrome, ARDS, Sequential organ failure assessment, SOFA

Posted Date: August 5th, 2020

DOI: https://doi.org/10.21203/rs.3.rs-52242/v1

License: (c) (i) This work is licensed under a Creative Commons Attribution 4.0 International License.

Read Full License 


\section{Abstract}

Background: For critically ill patients with coronavirus disease 2019 (COVID-19) who require intensive care unit (ICU) admission, mortality rates vary widely depending on many factors, among which hospital resources and clinical setting seem important. We sought to determine the outcome of critically ill patients admitted in the usual multidisciplinary ICUs of a big referral for COVID-19 tertiary-care hospital with adequate resources.

Methods: We performed a prospective observational study of all adult patients with COVID-19 consecutively admitted to four COVID-designated ICUs at Evangelismos Hospital, Athens, Greece, from March 11 to April 27, 2020.

Results: Among 50 critically ill patients, ICU and hospital mortality for the entire cohort was $32 \%(16 / 50)$, whereas $66 \%(33 / 50)$ of patients were discharged alive from the ICU and $2 \%(1 / 50)$ were still treated in the ICU until June 16, 2020. ICU and hospital mortality for those who received invasive mechanical ventilation was 39\% (16/41). Patients who eventually died had already increased risk of death on ICU admission, as suggested by the high values of the Acute Physiology and Chronic Health Evaluation II and Sequential Organ Failure Assessment scores, the presence of current malignancy and occurrence of cardiac arrest in $44 \%(7 / 16)$ of patients, and the general need for circulatory support by noradrenaline. Median $\mathrm{PaO}_{2} / \mathrm{FiO}_{2}$ on ICU admission for the entire cohort was $121 \mathrm{mmHg}$ [interquartile range (IQR), 86$171 \mathrm{mmHg}$ ] and most patients had moderate and severe acute respiratory distress syndrome (ARDS) according to the Berlin Definition. The primary cause of death of all patients was multi-organ failure, most commonly due to sepsis, whereas none died from refractory hypoxemia, neurologic dysfunction or withdrawal of life support. Hospital stay was long in patients who survived [median 24 days (IQR, 15-35 days)] and was frequently complicated by bacteremias [36\% (12/33)].

Conclusion: Severely ill COVID-19 patients with moderate and severe ARDS may have equal or even lower mortality rates compared to ARDS due to other causes, when they are admitted in general ICUs with experienced and adequate staff without limitations in hospital resources, where established ARDS therapies are used.

\section{Background}

The outbreak of coronavirus disease 2019 (COVID-19) emerged in China in December 2019 (1) and rapidly spread worldwide. The major clinical complication in patients with COVID-19 is acute respiratory distress syndrome (ARDS) requiring intensive care unit (ICU) admission. For those patients needing care in an ICU, mortality rates as high as 49 to $97 \%$ have been reported (2-9). However, there is report of lower mortality rates (30.9\% for all patients admitted in the ICU and $35.7 \%$ for intubated patients) in areas where ICU capacity enabled the timely admission of all COVID-19 patients requiring critical care to a traditional ICU (10). 
In Greece, the number of patients with confirmed COVID-19 and those who required hospital and ICU admission was rather limited, thus not overwhelming hospital resources (11). Therefore, we considered pertinent to investigate the outcome of critically ill patients with COVID-19 admitted to the general ICUs of a big referral for COVID-19 tertiary-care hospital with adequate resources.

\section{Methods}

\section{Patients and Setting}

This is a prospective observational study of all adult patients with COVID-19 consecutively admitted to four COVID-designated ICUs at Evangelismos Hospital, Athens, Greece, from March 11 to April 27, 2020. COVID-19 status was based upon positive severe acute respiratory syndrome coronavirus 2 real-time reverse transcriptase-polymerase chain reaction assay of nasopharyngeal-swab specimens. The study was approved by the hospital institutional review boards. Informed consent was waived.

During the study period, all patients with COVID-19 who required critical care were timely admitted to a COVID-ICU due to adequate ICU capacity. Additionally, all patients admitted to COVID-ICUs were treated by standard (i.e., pre-COVID) multidisciplinary ICU care teams with usual ICU staffing ratios. There was no lack of ventilators or other respiratory support devices, dialysis machines, medications, or other critical care supplies including personal protective equipment. Although there was an institutional recommendation against the routine use of non-invasive positive pressure mechanical ventilation, the routine use of high-flow nasal cannula oxygen therapy was not limited.

Clinical management occurred at the discretion of the critical-care trained attending physician. The management of mechanically ventilated patients was according to ARDS treatment guidelines $(12,13)$, including prone ventilation and conservative fluid management. Briefly, tidal volumes of approximately $6 \mathrm{ml} / \mathrm{kg}$ predicted body weight and a respiratory rate adjusted to maintain arterial $\mathrm{pH}$ above 7.30 were applied, targeting plateau pressure of less than $30 \mathrm{~cm} \mathrm{H}_{2} \mathrm{O}$. Positive end-expiratory pressure (PEEP) was usually set according to the lower PEEP / higher $\mathrm{FiO}_{2}$ ARDS network table (12) titrating for the best tidal respiratory system compliance.

\section{Data Collection and Definitions}

Data was collected through June 16, 2020. All patient data, clinical and laboratory, as well as ventilator settings and measurements were prospectively collected along with the ongoing pandemic. ARDS was defined according to the Berlin Definition criteria (14). Severity of illness was assessed by the Acute Physiology and Chronic Health Evaluation (APACHE) II (15) and Sequential Organ Failure Assessment (SOFA) (16) scores. The severity of acute pulmonary damage in mechanically ventilated patients was graded by using the Lung Injury Score (17). Respiratory system compliance was computed as the tidal volume divided by the difference between plateau pressure and total PEEP. The last difference constituted the driving pressure. The evolution of organ dysfunction during the ICU stay was evaluated by calculating the SOFA score at admission and on ICU days 3, 5, 10, 14 and 21. 


\section{Statistical Analysis}

Quantitative data is reported as median and interquartile range (IQR). Non-parametric statistics were applied. Comparisons between patients who survived versus those who died in the ICU were performed by using the Mann-Whitney $U$ test. Differences between these two groups of patients in qualitative variables were assessed by Chi-square or Fisher's exact test when appropriate. Differences in group data for related samples were evaluated by the Friedman's ANOVA by ranks; when significant differences were found, post-hoc comparisons were performed by using the Wilcoxon matched-pair test. The SPSS statistical program (version 10, Chicago, IL, USA) was used for data analysis. Statistical significance was defined as a two-tailed $p$ value of $<0.05$.

\section{Results}

\section{Patient Characteristics and ICU admission}

During the study period 50 adults with COVID-19 infection were critically ill and admitted to the ICUs of our institution (Fig. 1A). Demographics and data on ICU admission are summarized in Table 1. The median patient age was 64 years (IQR, 58-72) and 24 patients (48.0\%) were 65 years or older. The majority of patients were Caucasian (47 [94.0\%]). Hypertension was the most common comorbidity (14 [28.0\%]), followed by diabetes (9 [18.0\%]). Five patients (10.0\%) had hematologic malignancy, whereas 18 patients (36.0\%) had at least two comorbidities. 
Table 1

Demographics and clinical and laboratory data on ICU admission of patients with COVID-19a

\begin{tabular}{|c|c|c|c|c|}
\hline & $\begin{array}{l}\text { All } \\
(\mathrm{n}=50)^{b}\end{array}$ & $\begin{array}{l}\text { Survived } \\
\text { ICU } \\
(n=33)\end{array}$ & $\begin{array}{l}\text { Died in ICU } \\
(n=16)\end{array}$ & $p^{c}$ \\
\hline Age, years [median (IQR)] & $64(58-72)$ & $61(55-71)$ & $70(60-77)$ & 0.065 \\
\hline$\leq 64$ & $26(52.0)$ & $20(60.6)$ & 5 (31.3) & 0.054 \\
\hline$\geq 65$ & $24(48.0)$ & $13(39.4)$ & $11(68.7)$ & \\
\hline Gender, male & $38(76.0)$ & $25(75,8)$ & $12(75.0)$ & 0.954 \\
\hline Race & $47(94.0)$ & $30(90.9)$ & $16(100)$ & 0.542 \\
\hline Caucasian & $3(6.0)$ & $3(9.1)$ & $0(0)$ & \\
\hline \multicolumn{5}{|l|}{ Asian } \\
\hline Comorbidity & $14(28.0)$ & $9(27.3)$ & $4(25.0)$ & 0.866 \\
\hline Hypertension & $9(18.0)$ & $6(18.2)$ & $3(18.8)$ & 0.962 \\
\hline Diabetes mellitus & $6(12.0)$ & $3(9.1)$ & $3(18.8)$ & 0.333 \\
\hline Cardiovascular $^{d}$ & $5(10.0)$ & $1(3.0)$ & $4(25.0)$ & 0.034 \\
\hline Malignancy ${ }^{e}$ & $5(10.0)$ & $3(9.1)$ & $2(12.5)$ & 0.712 \\
\hline Obesity $f$ & $4(8.0)$ & $2(6.1)$ & $2(12.5)$ & 0.440 \\
\hline Chronic luna disease & $1(2.0)$ & $0(0)$ & $1(6.3)$ & 0.327 \\
\hline Chronic renal failure & $18(36.0)$ & $11(33.3)$ & $7(43.8)$ & 0.478 \\
\hline Comorbidities $(\geq 2)$ & & & & \\
\hline $\begin{array}{l}\text { Time from symptom onset to ICU admission, } \\
\text { days [median (IQR)] }\end{array}$ & $9(8-11)$ & $10(8-12)$ & $8(8-10.5)$ & 0.352 \\
\hline
\end{tabular}

ICU, intensive care unit; IQR, interquartile range; BMI, body mass index; APACHE, Acute Physiology and Chronic Health Evaluation (16); SOFA, Sequential Organ Failure Assessment (17); $\mathrm{PaO}_{2}$, partial pressure of arterial oxygen; $\mathrm{FiO}_{2}$, fraction of inspired oxygen; $\mathrm{PaCO}_{2}$, partial pressure of arterial carbon dioxide; CT, computed tomography; PEEP, positive end-expiratory pressure; LIS, Lung Injury Score (18).

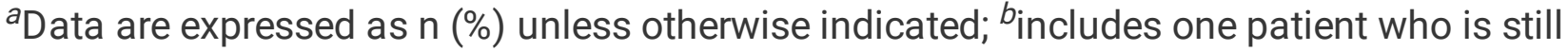
mechanically ventilated in the ICU; ${ }^{c}$ Mann-Whitney $U$ test and $X^{2}$ with Yates correction or Fisher exact test comparing those who survived versus died in the ICU; ${ }^{d}$ coronary artery disease and/or congestive heart failure; elymphoma, chronic lymphoid leukemia and acute myeloid leukemia had 3,1 and 1 patients, respectively ( 3 patients were under chemotherapy); ${ }_{\text {defined }}$ as $\mathrm{BMI}>30 \mathrm{~kg} / \mathrm{m}^{2} ; g_{\text {upper limit }}$ of normal $0.5 \mathrm{mg} / \mathrm{dL}$; ${ }^{h}$ upper limit of normal $0.1 \mathrm{ng} / \mathrm{mL}$; ${ }^{i}$ upper limit of normal $0.3 \mu \mathrm{g} / \mathrm{mL}$; ${ }^{j}$ upper limit of normal $14 \mathrm{pg} / \mathrm{mL}$; ${ }^{\text {refers to }} 41$ patients who received invasive mechanical ventilation. 


\begin{tabular}{|lllll|}
\hline & All & $\begin{array}{l}\text { Survived } \\
\text { ICU } \\
(\mathbf{n = 5 0})^{\boldsymbol{b}}\end{array}$ & $\begin{array}{l}\text { Died in ICU } \\
(\mathbf{n = 3 3 )}\end{array}$ & $p^{c}$ \\
\hline APACHE II score, median (IQR) & $12(8-17)$ & $9(6-12)$ & $20(17-26)$ & $<$ \\
\hline SOFA score, median (IQR) & $7(3-9)$ & $6(2-7)$ & $11(9-13)$ & $<$ \\
\hline
\end{tabular}

ICU, intensive care unit; IQR, interquartile range; BMI, body mass index; APACHE, Acute Physiology and Chronic Health Evaluation (16); SOFA, Sequential Organ Failure Assessment (17); $\mathrm{PaO}_{2}$, partial pressure of arterial oxygen; $\mathrm{FiO}_{2}$, fraction of inspired oxygen; $\mathrm{PaCO}_{2}$, partial pressure of arterial carbon dioxide; CT, computed tomography; PEEP, positive end-expiratory pressure; LIS, Lung Injury Score (18).

${ }^{a}$ Data are expressed as $\mathrm{n}(\%)$ unless otherwise indicated; $b_{\text {includes one patient who is still }}$ mechanically ventilated in the ICU; ${ }^{c}$ Mann-Whitney $U$ test and $X^{2}$ with Yates correction or Fisher exact test comparing those who survived versus died in the ICU; ${ }^{d}$ coronary artery disease and/or congestive heart failure; elymphoma, chronic lymphoid leukemia and acute myeloid leukemia had 3,1 and 1 patients, respectively ( 3 patients were under chemotherapy); ${ }^{f}$ defined as BMI $>30 \mathrm{~kg} / \mathrm{m}^{2} ; g_{\text {upper limit }}$ of normal $0.5 \mathrm{mg} / \mathrm{dL}$; ${ }^{h}$ upper limit of normal $0.1 \mathrm{ng} / \mathrm{mL}$; 'iupper limit of normal $0.3 \mu \mathrm{g} / \mathrm{mL}$; ${ }^{j}$ upper limit of normal $14 \mathrm{pg} / \mathrm{mL}$; ${ }^{\text {refers to }} 41$ patients who received invasive mechanical ventilation. 


\begin{tabular}{|c|c|c|c|c|}
\hline & $\begin{array}{l}\text { All } \\
(n=50)^{b}\end{array}$ & $\begin{array}{l}\text { Survived } \\
\text { ICU } \\
(n=33)\end{array}$ & $\begin{array}{l}\text { Died in ICU } \\
(n=16)\end{array}$ & $p^{c}$ \\
\hline Laboratory data, median (IQR) & $\begin{array}{l}7.6(5.9- \\
11.2)\end{array}$ & $\begin{array}{l}7.3(5.9- \\
10.8)\end{array}$ & $\begin{array}{l}9.5(6.0- \\
17.3)\end{array}$ & $\begin{array}{l}0.386 \\
0.855\end{array}$ \\
\hline Leukocyte count, $x 10^{9} / \mathrm{L}$ & $\begin{array}{l}0.88(0.61- \\
1.13)\end{array}$ & $\begin{array}{l}0.88(0.65- \\
1.13)\end{array}$ & $\begin{array}{l}0.93(0.48- \\
1.08)\end{array}$ & 0.553 \\
\hline Platelet count, $\times 10^{9} / \mathrm{L}$ & $\begin{array}{l}233(193- \\
318)\end{array}$ & $\begin{array}{l}233(200- \\
322)\end{array}$ & $\begin{array}{l}263(211- \\
329)\end{array}$ & \\
\hline C-reactive protein, $\mathrm{mg} / \mathrm{dL}^{g}$ & $\begin{array}{l}14.2(7.7- \\
24.6)\end{array}$ & $\begin{array}{l}13.6(7.7- \\
20.3)\end{array}$ & $\begin{array}{l}21.7(9.6- \\
31.9)\end{array}$ & $\begin{array}{l}0.503 \\
0.345\end{array}$ \\
\hline Procalcitonine, $\mathrm{ng} / \mathrm{mL}^{h}$ & $\begin{array}{l}0.43(0.12- \\
0.94\end{array}$ & $\begin{array}{l}0.34(0.15- \\
0.66)\end{array}$ & $\begin{array}{l}1.41(0.11- \\
1.52)\end{array}$ & \multirow{2}{*}{$\begin{array}{l}0.987 \\
0.316 \\
\mathbf{0 . 0 3 8}\end{array}$} \\
\hline D-dimer, $\mu \mathrm{g} / \mathrm{mL}^{i}$ & $\begin{array}{l}835(353- \\
1882)\end{array}$ & $\begin{array}{l}835(576- \\
1710)\end{array}$ & $\begin{array}{l}1509(479- \\
1740)\end{array}$ & \\
\hline Lactate dehydrogenase, IU/L & $\begin{array}{l}1.23(0.52- \\
2.46)\end{array}$ & $\begin{array}{l}1.30(0.59- \\
2.43)\end{array}$ & $\begin{array}{l}1.17(0.45- \\
2.17)\end{array}$ & \multirow{3}{*}{$\begin{array}{l}0.123 \\
0.012 \\
< \\
0.001\end{array}$} \\
\hline $\begin{array}{l}\text { Albumin, g/dL } \\
\text { Creatinine, mg/dL }\end{array}$ & $\begin{array}{l}471(403- \\
611)\end{array}$ & $\begin{array}{l}454(363- \\
604)\end{array}$ & $\begin{array}{l}509(428- \\
574)\end{array}$ & \\
\hline Troponin $\mathrm{T}, \mathrm{pg} / \mathrm{mL}^{j}$ & $\begin{array}{l}3.2(3.0- \\
3.6)\end{array}$ & $\begin{array}{l}3.3(3.1- \\
3.7)\end{array}$ & $\begin{array}{l}3.0(2.8- \\
3.4)\end{array}$ & \\
\hline \multirow{3}{*}{ Lactate, $\mathrm{mmol} / \mathrm{L}$} & $\begin{array}{l}0.9(0.7- \\
1.1)\end{array}$ & $\begin{array}{l}0.8(0.7- \\
1.0)\end{array}$ & \multicolumn{2}{|l|}{$\begin{array}{l}1.1(0.7- \\
1.8)\end{array}$} \\
\hline & $15(10-52)$ & $12(8-20)$ & \multicolumn{2}{|l|}{$31(14-85)$} \\
\hline & $\begin{array}{l}1.3(1.0- \\
1.8)\end{array}$ & $\begin{array}{l}1.0(0.9- \\
1.3)\end{array}$ & $\begin{array}{l}1.8(1.3- \\
2.1)\end{array}$ & \\
\hline Bilateral infiltrates on chest $\mathrm{x}$-ray/CT & $49(98)$ & $32(97)$ & $16(100)$ & 1.000 \\
\hline \multicolumn{5}{|l|}{ Arterial blood gases, median (IQR) } \\
\hline \multicolumn{5}{|c|}{$\begin{array}{l}\text { ICU, intensive care unit; IQR, interquartile range; } \mathrm{BMI} \text {, body mass index; } \mathrm{APACHE} \text {, Acute Physiology and } \\
\text { Chronic Health Evaluation (16); SOFA, Sequential Organ Failure Assessment (17); } \mathrm{PaO}_{2} \text {, partial } \\
\text { pressure of arterial oxygen; } \mathrm{FiO}_{2} \text {, fraction of inspired oxygen; } \mathrm{PaCO}_{2} \text {, partial pressure of arterial carbon } \\
\text { dioxide; } \mathrm{CT} \text {, computed tomography; PEEP, positive end-expiratory pressure; LIS, Lung Injury Score (18). }\end{array}$} \\
\hline \multicolumn{5}{|c|}{ 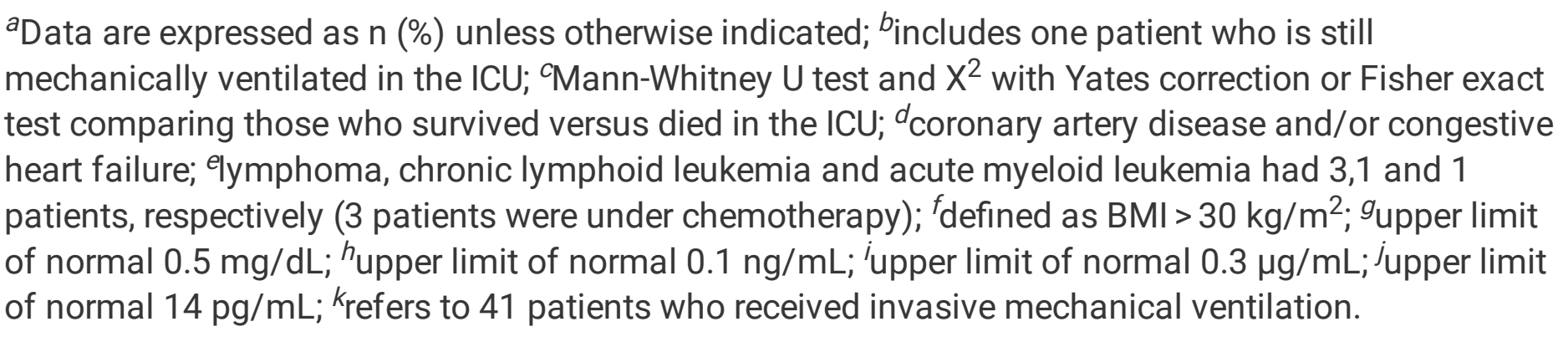 } \\
\hline
\end{tabular}




\begin{tabular}{|c|c|c|c|c|}
\hline & $\begin{array}{l}\text { All } \\
(n=50)^{b}\end{array}$ & $\begin{array}{l}\text { Survived } \\
\text { ICU } \\
(n=33)\end{array}$ & $\begin{array}{l}\text { Died in ICU } \\
(n=16)\end{array}$ & $p^{c}$ \\
\hline $\mathrm{PaO}_{2} / \mathrm{FiO}_{2}, \mathrm{mmHg}$ & $\begin{array}{l}121(86- \\
171)\end{array}$ & $\begin{array}{l}119(89- \\
157)\end{array}$ & $\begin{array}{l}123(75- \\
243)\end{array}$ & 0.790 \\
\hline $\mathrm{PaCO}_{2}, \mathrm{mmHg}$ & $40(33-45)$ & $36(29-42)$ & $44(42-50)$ & 0.002 \\
\hline $\mathrm{pH}$ & $\begin{array}{l}7.39(7.32- \\
7.44)\end{array}$ & $\begin{array}{l}7.42(7.35- \\
7.45)\end{array}$ & $\begin{array}{l}7.30(7.25- \\
7.36)\end{array}$ & 0.006 \\
\hline \multicolumn{5}{|c|}{$\begin{array}{l}\text { Respiratory parameters at day of intubation, } \\
\text { median (IQR) }\end{array}$} \\
\hline PEEP, $\mathrm{cmH}_{2} \mathrm{O}$ & $14(12-16)$ & $14(12-17)$ & $13(10-16)$ & 0.436 \\
\hline Plateau pressure, $\mathrm{cmH}_{2} \mathrm{O}$ & $27(25-29)$ & $27(26-29)$ & $25(24-29)$ & 0.413 \\
\hline Driving pressure, $\mathrm{cmH}_{2} \mathrm{O}$ & $13(11-15)$ & $12(10-14)$ & $13(12-15)$ & 0.384 \\
\hline Static compliance, $\mathrm{mL} / \mathrm{cmH}_{2} \mathrm{O}$ & $40(32-50)$ & $40(33-50)$ & $39(33-42)$ & 0.683 \\
\hline LIS & $\begin{array}{l}2.7(2.5- \\
3.2)\end{array}$ & $\begin{array}{l}2.7(2.5- \\
3.2)\end{array}$ & $\begin{array}{l}2.8(2.5- \\
3.5)\end{array}$ & 0.730 \\
\hline Noradrenaline & $39(78)$ & $21(64)$ & $16(100)$ & 0.004 \\
\hline \multicolumn{5}{|c|}{$\begin{array}{l}\text { ICU, intensive care unit; IQR, interquartile range; } \mathrm{BMI} \text {, body mass index; } \mathrm{APACHE} \text {, Acute Physiology and } \\
\text { Chronic Health Evaluation (16); SOFA, Sequential Organ Failure Assessment (17); } \mathrm{PaO}_{2} \text {, partial } \\
\text { pressure of arterial oxygen; } \mathrm{FiO}_{2} \text {, fraction of inspired oxygen; } \mathrm{PaCO}_{2} \text {, partial pressure of arterial carbon } \\
\text { dioxide; } \mathrm{CT} \text {, computed tomography; PEEP, positive end-expiratory pressure; LIS, Lung Injury Score (18). }\end{array}$} \\
\hline \multicolumn{5}{|c|}{ 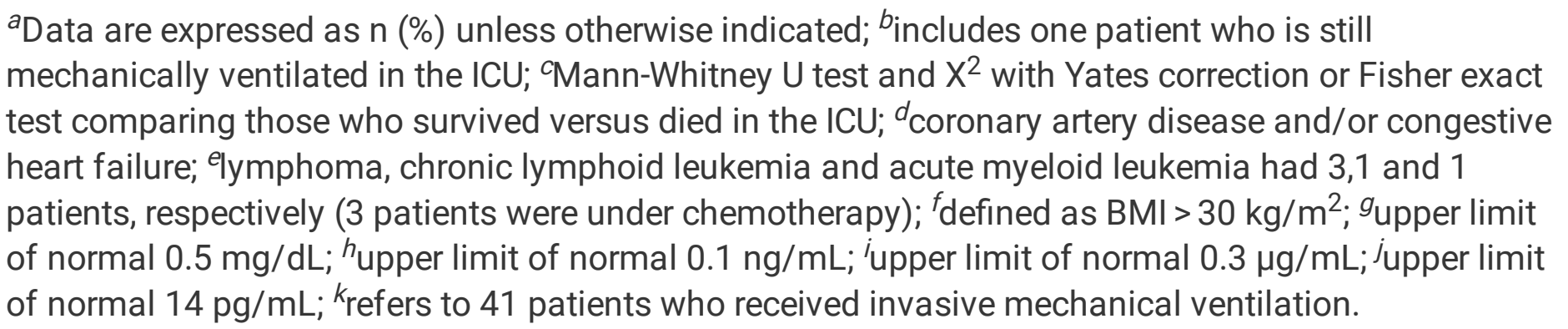 } \\
\hline
\end{tabular}

The median time from symptom onset to hospital admission was 7 days (IQR, 5.0-8.5 days). Twentythree patients (46\%) were admitted to the ICU within less than 24 hours after hospital admission. The remaining 27 patients (54\%) had median hospitalization time in general wards prior to ICU admission 2 days (IQR, $1-4$ days).

On ICU admission, the median APACHE II score was 12 (IQR, 8-17), and the median SOFA score was 7 (IQR, 3-9). Bilateral lung infiltrates were shown in 49 patients (98\%), whereas the median $\mathrm{PaO}_{2} / \mathrm{FiO}_{2}$ was 
$121 \mathrm{mmHg}$ (IQR, 86-171 mmHg). Thirty-nine patients (78\%), all under invasive mechanical ventilation, were receiving noradrenaline for circulatory support. In none of the patients was the respiratory viral panel positive for a different viral infection or the respiratory samples positive for bacteria.

Invasive mechanical ventilation was applied in 41 patients (82\%) (Fig. 1B). On the day of intubation, median static respiratory system compliance was $40 \mathrm{~mL} / \mathrm{cmH}_{2} \mathrm{O}\left(\mathrm{IQR}, 32-50 \mathrm{~mL} / \mathrm{cmH}_{2} \mathrm{O}\right)$ and median Lung Injury Score was 2.7 (IQR, 2.5-3.2) (ARDS is identified when Lung Injury Score is higher than 2.5 [18]).

\section{Interventions in the ICU}

Interventions in the ICU are included in Table 2. A total of 14 patients $(28 \%)$ received high-flow nasal cannula whereas only 2 patients (4\%) were treated with non-invasive mechanical ventilation. Among those who received invasive mechanical ventilation, the median duration of mechanical ventilation was 13 days (IQR, 9-33 days), while 26 (52\%) required neuromuscular blockade therapy for at least 24 hours, and prone position was used as rescue therapy in 6 patients (12\%). Thirty-four patients (68\%) required noradrenaline as vasopressor support for shock and 13 patients $(26 \%)$ needed continuous renal replacement therapy. Most of the patients received hydroxychloroquine for at least 5 days. 
Table 2

Interventions in the ICU and outcomes ${ }^{a}$

\begin{tabular}{|c|c|c|c|c|}
\hline & $\begin{array}{l}\text { All } \\
(\mathrm{n}=50)^{b}\end{array}$ & $\begin{array}{l}\text { Survived ICU } \\
(n=33)\end{array}$ & $\begin{array}{l}\text { Died in ICU } \\
(n=16)\end{array}$ & $p^{c}$ \\
\hline High-flow nasal cannula & $14(28)$ & $13(39)$ & $1(6)$ & 0.019 \\
\hline Non-invasive mechanical ventilation & $2(4)$ & $2(6)$ & $0(0)$ & 0.551 \\
\hline Invasive mechanical ventilation (IMV) & $41(82)$ & $24(73)$ & $16(100)$ & 0.021 \\
\hline Time to intubation, median (IQR) ${ }^{d}$ & $2(0-3)$ & $2(0-4)$ & $1(0-3)$ & 0.249 \\
\hline IMV days, median (IQR) & $13(9-33)$ & $13(10-32)$ & $14(7-27)$ & 0.847 \\
\hline Neuromuscular blockade & $26(52)$ & $14(42)$ & $12(75)$ & 0.066 \\
\hline Prone position & $6(12)$ & $4(12)$ & $2(13)$ & 0.969 \\
\hline Noradrenaline & $34(68)$ & $17(52)$ & $16(100)$ & $<0.001$ \\
\hline Noradrenaline days, median (IQR) & $6(2-13)$ & $4(1-8)$ & $11(5-15)$ & 0.009 \\
\hline Renal replacement therapy & $13(26)$ & $4(12)$ & $8(50)$ & 0.003 \\
\hline $\begin{array}{l}\text { Renal replacement therapy days, } \\
\text { median (IQR) }\end{array}$ & $15(11-24)$ & $19(15-24)$ & $15(9-26)$ & 0.570 \\
\hline Selected inpatient medications & $44(88)$ & $29(88)$ & $14(88)$ & 0.969 \\
\hline Hydroxychloroquine & $38(76)$ & $26(79)$ & $12(75)$ & 0.765 \\
\hline Azithromycin & $18(36)$ & $13(39)$ & $5(31)$ & 0.811 \\
\hline Lopinavir / Ritonavir & $5(10)$ & $3(9)$ & $2(13)$ & 0.893 \\
\hline Anti-Interleukin-6 antibody & $3(6)$ & $1(3)$ & $1(6)$ & 0.813 \\
\hline Remdesivir (or placebo) & $5(10)$ & $2(6)$ & $3(19)$ & 0.382 \\
\hline Glucocorticoids & & & & \\
\hline
\end{tabular}

ICU, intensive care unit; IQR, interquartile range; NA, not applicable.

${ }^{a}$ Data are expressed as $\mathrm{n}(\%)$ unless otherwise indicated; $b_{\text {includes one patient who is still }}$ mechanically ventilated in the ICU; ${ }^{c}$ Mann-Whitney $U$ test and $X^{2}$ with Yates correction or Fisher exact test comparing those who survived versus died in the ICU; ${ }^{d}$ refers to 41 patients who received invasive mechanical ventilation; ${ }^{e}$ among patients who did not have a tracheostomy; ${ }^{f}$ patients who survived and were discharged alive from the ICU $(n=24)$ were the sum of patients who were successfully extubated $(n=17)$ and those who had a tracheostomy $(n=7)$. 


\begin{tabular}{|c|c|c|c|c|}
\hline & $\begin{array}{l}\text { All } \\
(n=50)^{b}\end{array}$ & $\begin{array}{l}\text { Survived ICU } \\
(n=33)\end{array}$ & $\begin{array}{l}\text { Died in ICU } \\
(n=16)\end{array}$ & $p^{c}$ \\
\hline Outcomes & $50(29-88)$ & $50(29-84)$ & $50(30-88)$ & NA \\
\hline Follow-up, days [median (range)] & $17(41)$ & $17(71)^{f}$ & $0(0)$ & NA \\
\hline Successful extubation ${ }^{d, e}$ & $9(5-14)$ & $9(5-14)$ & NA & 0.942 \\
\hline Time to successful extubation, days & $12(29)$ & $7(29)^{f}$ & $4(25)$ & 0.968 \\
\hline [median (IQR) $)^{d, e}$ & $22(19-25)$ & $21(19-26)$ & $22(18-26)$ & 0.847 \\
\hline Tracheostomy $^{d}$ & $13(9-33)$ & $13(10-32)$ & $14(7-27)$ & 0.417 \\
\hline Time to tracheostomy, days & $14(9-31)$ & $15(10-30)$ & $14(7-27)$ & $<0.001$ \\
\hline$[\text { median (IQR) }]^{d}$ & $21(10-32)$ & $24(15-35)$ & $14(7-27)$ & NA \\
\hline $\begin{array}{l}\text { Mechanical ventilation days, } \\
\text { median (IQR) }^{d}\end{array}$ & $\begin{array}{l}12(24) \\
12(29)\end{array}$ & $\begin{array}{l}\text { NA } \\
\text { NA }\end{array}$ & $\begin{array}{l}\text { NA } \\
\text { NA }\end{array}$ & NA \\
\hline ICU days, median (IQR) & & & & \\
\hline Hospital days, median (IQR) & & & & \\
\hline 28-day mortality & & & & \\
\hline 28-day mortality in ventilated ${ }^{d}$ & & & & \\
\hline \multicolumn{5}{|c|}{ ICU, intensive care unit; IQR, interquartile range; NA, not applicable. } \\
\hline \multicolumn{5}{|c|}{$\begin{array}{l}{ }^{a} \text { Data are expressed as } \mathrm{n}(\%) \text { unless otherwise indicated; }{ }^{b} \text { includes one patient who is still } \\
\text { mechanically ventilated in the ICU; }{ }^{C} \text { Mann-Whitney } U \text { test and } X^{2} \text { with Yates correction or Fisher exact } \\
\text { test comparing those who survived versus died in the ICU; }{ }^{d} \text { refers to } 41 \text { patients who received invasive } \\
\text { mechanical ventilation; }{ }^{e} \text { among patients who did not have a tracheostomy; }{ }^{f} \text { patients who survived } \\
\text { and were discharged alive from the ICU }(n=24) \text { were the sum of patients who were successfully } \\
\text { extubated }(n=17) \text { and those who had a tracheostomy }(n=7) \text {. }\end{array}$} \\
\hline
\end{tabular}

\section{ICU Outcomes}

ICU outcomes are displayed in Fig. 1 and summarized in Table 2. As of data censoring on June 16, 2020, median patient follow-up was 50 days (range 29-88) (Table 2). Among 50 patients admitted in the ICU, 16 died (32.0\%), 33 patients were discharged alive from the ICU (66.0\%), and 1 patient remained in the ICU (2.0\%) still receiving invasive mechanical ventilation; hospital mortality was $32.0 \%$ as well $(16 / 50)$ (Fig. 1A), whereas 28-day mortality was 24\% (12/50) (Table 2). Among 41 patients who received invasive mechanical ventilation, ICU and hospital mortality was 39.0\% (16/41) (Fig. 1B); 24 patients were 
discharged alive from the ICU (58.6\%), 17 of them after a successful extubation and the remaining 7 patients with a tracheostomy in place (Table 2). Twenty-eight-day mortality was $29 \%$ (12/41) (Table 2).

Patients who died had a higher proportion of current malignancy, and on ICU admission, had higher APACHE II and SOFA scores, higher blood troponin $T$ and lactate values, and were receiving noradrenaline for vasopressor circulatory support more frequently than those who survived (Table 1). Notably, among patients who died, 3 (19\%) had suffered cardiac arrest during endotracheal intubation. Compared to those who survived, patients who died in the ICU rarely received high-flow nasal cannula oxygen therapy, and more commonly required invasive mechanical ventilation, noradrenaline infusion and continuous renal replacement therapy for renal failure management (Table 2). Duration of hospital stay was longer in patients who survived compared to those who died in ICU (Table 2).

SOFA score in patients who died was higher than in those who survived not only on ICU admission, but also on days 3-21 ( $p<0.01$ for all days) (Fig. 2). This difference could be attributed to higher cardiovascular and renal components of SOFA score in patients who died compared to those who survived ICU (data not shown). SOFA score in patients who survived was not significantly different from ICU admission to day 21. In contrast, SOFA score in patients who died did differ in the course of their ICU stay; post-hoc analysis showed that their SOFA scores on days 10, 15 and 21 were all significantly higher than on admission, day 3 or day 5 ( $p<0.05$ for all days) (Fig. 2). This difference was mainly due to the higher renal component of SOFA score (data not shown). The primary cause of death of all patients was multi-organ failure most commonly due to sepsis; none died from refractory hypoxemia, neurologic dysfunction or withdrawal of life support (18). Thrombotic events (i.e., deep venous thrombosis) occurred in 3 patients (6\%) (2 patients survived and 1 died); in 1 patient who eventually survived, deep venous thrombosis was complicated by intermediate-risk pulmonary embolism. ICU-acquired bacteremia was detected in 12 patients who survived $(36 \%)$ versus 4 patients who died $(25 \%)(p=0.637)$. Median number of bacteremias in patients who survived was 2.5 (IQR, 1.0-4.5) versus 1.5 (IQR, 1.0-4.0) in patients who died $(p=0.770)$. Klebsiella pneumoniae, Acinetobacter baumannii, and Enterococcus faecalis were the most frequent bacteria, and were isolated in $6(18 \%), 6(18 \%)$ and 5 patients $(15 \%)$, respectively, in patients who survived compared to $3(19 \%), 2(13 \%)$ and 2 patients $(13 \%)$, respectively, in patients who died $(p=0.729-0.926)$.

\section{Discussion}

The main findings of the present study were: i) ICU and hospital mortality were $32 \%$ and $39 \%$ for the entire cohort of patients admitted to the ICU and for those who received invasive mechanical ventilation, respectively; ii) 28 -day mortality was $24 \%$ and $29 \%$ for the whole group of patients admitted in the ICU and for those who required invasive mechanical ventilation, respectively; iii) patients who eventually died had already increased risk of death even on ICU admission, as suggested by the high values of APACHE II and SOFA scores, the presence of current malignancy and occurrence of cardiac arrest, and general need for circulatory support with noradrenaline; iv) the primary cause of death of all patients was multi-organ failure most commonly due to sepsis, whereas none died from refractory hypoxemia, neurologic 
dysfunction or withdrawal of life support; and v) hospital stay was long in patients who survived, frequently complicated by bacteremias.

ICU and hospital mortality of $32 \%$ for overall ICU admissions observed in the present study was similar to that recently reported by Auld et al (10) in a large cohort of critically ill patients $(n=217)$ admitted in COVID-designated ICUs of 3 hospitals in Atlanta, Georgia, showing $28.6 \%$ ICU mortality and $31 \%$ hospital mortality. Moreover, ICU and hospital mortality of $39 \%$ for the approximately four-fifths of patients in our cohort who received invasive mechanical ventilation was also similar to that of Auld et al (10), reporting $33.9 \%$ ICU mortality and $35.7 \%$ hospital mortality. Notably, only $2.4 \%$ of patients in our cohort and $4.8 \%$ of patients in the previous study (10) still remained on the ventilator at the time of the report, and there was ample follow-up time (median 50 days, range 29-88 days, in our group of patients) (Table 2). Both patient cohorts, i.e., that of ours and that of Auld et al (10), seem comparable in terms of lung disease and patient illness severity, at least on ICU admission. Indeed, median $\mathrm{PaO}_{2} / \mathrm{FiO}_{2}$ was $121 \mathrm{mmHg}(\mathrm{IQR}$, 86-171 mmHg) with median PEEP $14 \mathrm{cmH}_{2} \mathrm{O}\left(\mathrm{IQR}, 12-16 \mathrm{cmH}_{2} \mathrm{O}\right)$ in our cohort versus median $\mathrm{PaO}_{2} / \mathrm{FiO}_{2}$ of $132 \mathrm{mmHg}$ (IQR, 100-178 mmHg) (PEEP level was not reported) in the previous study (10); median SOFA score was 7 in both studies. Most of our patients had moderate and severe ARDS according to the ARDS Berlin Definition (14); the definition of ARDS was also fulfilled in most of our mechanically ventilated patients on the day of intubation, as the median Lung Injury Score was 2.7 (IQR, 2.5-3.2) (17) (Table 1). Our data, as well as that of Auld et al (10), provide evidence that mortality rates of severely ill COVID-19 patients with ARDS may be comparable or even lower to those reported in ARDS of different etiologies $(19,20)$ including Influenza A $(21,22)$. Indeed, hospital mortality for patients with moderate ARDS was $40.3 \%$ and for those with severe ARDS was $46.1 \%$ (19), whereas overall pooled mortality rate of all ARDS studies included in a comprehensive literature review was $43 \%$ (20). Moreover, mortality rate was $41.4 \%$ (21) and $46 \%$ (22) among ICU patients with Influenza A pneumonia, most of whom had ARDS.

Our findings differ from those of earlier studies in ICU patients with COVID-19 reporting overall mortality rates $49-62 \%(2,7,8)$ and mortality rates as high as $66-97 \%$ among patients requiring invasive mechanical ventilation in China $(7,23,24)$. Similarly, mortality rate for our patients was substantially lower than that of subsequent reports from USA (4-6), showing mortality rates for patients requiring invasive mechanical ventilation ranging from $52 \%$ (5) and $67 \%$ (6) in the Seattle region to $88.1 \%$ in New York area (4), as well as from Italy demonstrating overall hospital mortality $53.4 \%$ for patients admitted in ICU (25). To interpret the observed differences in clinical outcomes, at least two general and/or local factors should be taken into account. Firstly and primarily, in our study all critically ill patients with COVID-19 were admitted to the ICU in time, because overwhelming stress on the healthcare system did not occur in our country (11). All these patients were admitted to preexisting multidisciplinary ICUs, were cared for by critical care teams with experience in the management of patients with severe acute respiratory failure at standard staffing ratios, and received full intensive care support, including renal replacement therapy. Secondly, the onset and peak of the COVID-19 pandemic in Greece occurred later than in many of the regions from earlier reports, including neighboring Italy (25). This delay gave time to 
create organizational arrangements, purchase equipment, train personnel, make consensus-driven clinical protocols, and distribute supplies across the healthcare system.

Lung disease on ICU admission was not worse in patients who died than in those who survived as suggested by lack of any difference in blood oxygenation, respiratory system compliance and Lung Injury Score values; the same ventilatory protocol was also applied in both groups as implied by the presence of no difference in PEEP, plateau pressure and driving pressure levels (Table 1). Moreover, lung disease severity on admission and ICU days 3-21 did not contribute to the higher SOFA score in patients who died compared to those who survived (Fig. 2) and nobody died from refractory hypoxemia. The higher SOFA score on admission and ICU days 3-21 (Fig. 2) was mainly due to higher cardiovascular and renal components of SOFA score. Not surprisingly, patients who died compared to patients who survived, more commonly needed noradrenaline infusion for circulatory support on ICU admission (Table 1) and required noradrenaline use for shock treatment and continuous renal replacement therapy for renal failure management during ICU stay (Table 2).

We acknowledge the main limitation of this study, i.e., we reported findings and outcomes from a single center and included a rather limited number of patients compared to hundreds or thousands of patients incorporated in many studies from other countries with thousands victims of COVID-19 pandemic (2-4, 9, $23,25,26)$. However, our study included all consecutive patients admitted to four COVID-designated ICUs of the biggest referral center for COVID-19 in Greece and our study sample represents about $25 \%$ of the total number of patients admitted in Greek ICUs (27). Due to the relatively limited spread of the pandemic in Greece, our medical system was not overwhelmed, and this fact generated the purpose of the present study. Therefore, our findings might not be generalizable to different populations and medical systems, but might be relevant to other countries where the pandemic did not overwhelm the health system capacity.

\section{Conclusions}

Severely ill COVID-19 patients with moderate and severe ARDS may have equal or even lower mortality rates compared to ARDS due to other causes, when they are admitted in general ICUs with experienced and adequate staff without limitations in hospital resources, where established ARDS therapies $(12,13)$, including low tidal volume and conservative fluid management, are used. The reported hospital mortality rates of $32 \%$ overall and $39 \%$ for patients who received invasive mechanical ventilation could potentially be used as basis for future treatment protocols in similar clinical settings.

\section{Abbreviations}

COVID-19: Coronavirus disease 2019; ICU: Intensive care unit; ARDS: Acute respiratory distress syndrome; PEEP: Positive end-expiratory pressure; APACHE: Acute physiology and chronic health evaluation; SOFA: Sequential organ failure assessment; IQR: interquartile range 


\section{Declarations}

\section{Authors' contributions}

All authors have contributed to the final version of the manuscript as follows: CR, EM, SK, IS, DZ, EI, SM, TD, CV, PK, LD, AS, IS, VM, AA, PT, AK, PP, ZM, ED, TT, IP, PG, AS, AM, EM, AK, AS, EG, and AK contributed to patients' inclusion; CR, EM, SK, and IS contributed to data collection; CR, SM, IK, VP, and SZ contributed to data analysis; SZ made the statistical analysis; $\mathrm{CR}$, and SZ drafted and revised the manuscript. The authors read and approved the final manuscript.

\section{Acknowledgements}

We would like to extend our most profound thanks and gratitude to our colleagues in the First Department of Intensive Care Medicine, School of Medicine, National and Kapodistrian University of Athens, at 'Evangelismos' Hospital, and the Department of Anesthesiology and Unit for Infectious Diseases, at the same Hospital, who have worked so hard to provide excellent clinical care during this global pandemic. Our colleagues were: Paraskevi Athanasiou, Aikaterini Alexandropoulou, Dimitris Pistolas, Fofo Menti, Anastasia Karagiorgou, Konstantinos Tsakismenos, Sofia Kostourou, Stavroula Arkoudi, Vassiliki Tori, Georgios Gikas, Maria Sagani, Panagiota Dimopoulou, Eleni Stathatou, Marina Kalergi, Panagiota Oikonomopoulou, Aikaterini Takopoulou, Aikaterini Evodia, Aikaterini Michalopoulou, Mariana Kahri, Michalis Vitos, Konstantinos Giakoumidakis, and Theodoros Mikropoulos.

\section{Funding}

None

\section{Availability of data and materials}

The datasets used/or analyzed in the present study are available from the corresponding author on reasonable request.

\section{Ethics approval and consent to participate}

This study was approved by the ethical committee of 'Evangelismos' Hospital, Athens, Greece. The need of informed consent was waived because of the observational nature of the study.

\section{Consent for publication}

Not applicable

\section{Competing interests}

None 


\section{References}

1. Guan W, Ni Z, Hu Y, Liang W, Ou C, He J, et al. Clinical characteristics of coronavirus disease 2019 in China. N Engl J Med. 2020; 382(18):1708-1720.

2. Yang $X, Y u$ Y, Xu J, Shu H, Xia J, Liu H, et al. Clinical course and outcomes of critically ill patients with SARS-CoV-2 pneumonia in Wuhan, China: a single-centered, retrospective, observational study. Lancet Respir Med 2020; 8: 475-481.

3. Zhou F, Yu T, Du R, Fan G, Liu Y, Liu Z, et al. Clinical course and risk factors for mortality of adult inpatients with COVID-19 in Wuhan, China: a retrospective cohort study. Lancet. 2020;395:10541062.

4. Richardson S., Hirsch J., Narasimhan M., Crawford J., McGinn T., Davidson K., et al. Presenting characteristics, comorbidities, and outcomes among 5700 patients hospitalized with COVID-19 in the New York City Area. 2020;323:2052-2059.

5. Arentz M, YimE, Klaff L, Lokhandwala S, Riedo F, Chong M, et al. Characteristics and outcomes of 21 critically ill patients with COVID-19 inWashington State. JAMA. 2020;323:1612-1614.

6. Bhatraju P, Ghassemieh B, Nichols M, Kim R, Jerome K, Nalla A, et al. Covid-19 in Critically III Patients in the Seattle Region - Case Series. N Engl J Med 2020; 382:2012-2022.

7. Wu C, Chen X, Cai Y, Xia J, Zhou X, Xu S, et al: Risk factors associated with acute respiratory distress syndrome and death in patients with coronavirus disease 2019 pneumonia in Wuhan, China. JAMA Intern Med 2020; 180:1-11.

8. Wu Z, McGoogan JM. Characteristics of and important lessons from the coronavirus disease 2019 (COVID-19) outbreak in China: summary of a report of 72314 cases from the Chinese Center for Disease Control and Prevention. JAMA 2020; doi:10.1001/jama.2020.2648.

9. Wang Y, Lu X, Chen H, Chen T, Su N, Huang F, et al. Clinical course and outcomes of 344 intensive care patients with COVID-19. Am J Respir Crit Care Med. 2020;201:1430-1434.

10. Auld S, Caridi-Scheible M, Blum J, Robichaux C, Kraft C, Jacob J, et al. ICU and Ventilator Mortality Among Critically III Adults With Coronavirus Disease 2019. Crit Care Med 2020; doi: 10.1097/CCM.0000000000004457.

11. Moris D, Schizas Lockdown During COVID-19: The Greek Success. In Vivo 2020;34 Suppl 3:16951699.

12. Brower R, Matthay M, Morris A, Schoenfeld D, Thompson T, Taylor B, et al. Ventilation with lower tidal volumes as compared with traditional tidal volumes for acute lung injury and the acute respiratory distress syndrome. N Engl J Med. 2000;342:1301-1308.

13. Alhazzani W, Møller M, Arabi Y, Loeb M, Gong MN, Fan E, et al. Surviving sepsis campaign: guidelines on the management of critically ill. Intensive Care Med. 2020;46:854-87.

14. Ranieri V, Rubenfeld G, Thompson B, Ferguson N, Caldwell E, Fan E, Camporota L, et al. ARDS Definition Task Force. Acute respiratory distress syndrome: The Berlin definition. JAMA 2012;307:2526-2533. 
15. Knaus W, Draper E, Wagner D, Zimmerman J. APACHE II: a severity of disease classification system. Crit Care Med 1985;13: 818-829.

16. Vincent JL, de Mendonça A, Cantraine F, Moreno R, Takala J, Suter P,et al. Use of the SOFA score to assess the incidence of organ dysfunction/failure in intensive care units: results of a multicenter, prospective study. Working group on "sepsis-related problems" of the European Society of Intensive Care Medicine. Crit Care Med. 1998;26:1793-800.

17. Murray J, Matthay M, Luce J, Flick M. An Expanded definition of the adult respiratory distress syndrome. Am Rev Respir Dis 1988;138:720-7233.

18. Vincent JL, Taccone F. Understanding pathways to death in patients with COVID-19 Lancet Respir Med. 2020; 8: 430-432.

19. Bellani G, Laffey J, Pham T, Fan E, Brochard L, Esteban A, et al. LUNG SAFE Investigators; ESICM Trials Group: Epidemiology, patterns of care, and mortality for patients with acute respiratory distress syndrome in intensive care units in 50 countries. JAMA 2016;315:788-800.

20. Zambon M, Vincent JL. Mortality rates for patients with acute lung injury/ARDS have decreased over time. Chest 2008;133:1120-1127.

21. Dominguez-Cherit G, Lapinsky S, Macias A, Pinto R, Espinoza-Perez L, de la Torre A, et al. Critically ill patients with 2009 influenza A(H1N1) in Mexico. JAMA 2009;302:1880-1887.

22. Estenssoro E, Rios F, Apezteguia C, Reina R, Neira J, Ceraso D, et al. Pandemic 2009 influenza A in Argentina. Am J Respir Crit Care Med 2010;182:41-48.

23. Ruan Q, Yang K, Wang W, Jiang L, Song J. Clinical predictors of mortality due to COVID-19 based on an analysis of data of 150 patients from Wuhan, China. Intensive Care Med. 2020; doi: 10.1007/s00134-020-05991.

24. Phua J, Weng L, Ling L, Egi M, Lim CM, , Divatia JV, et al. Intensive care management of coronavirus disease 2019 (COVID-19): challenges and recommendations Lancet Respir Med. 2020; 8:506-517.

25. Grasselli G, Greco M, Zanella A, Albano G, Antonelli M, Bellani G, et al. Risk factors associated with mortality among patients with COVID-19 in Intensive Care Units in Lombardy, Italy. JAMA Intern Med. 2020; doi: 10.1001/jamainternmed.2020.3539.

26. Gupta S, Hayek S, Wang W, Chan L, Mathews K, Melamed M, et al. Factors associated with death in critically ill patients with coronavirus disease 2019 in the US. JAMA Internal Medicine; doi:10.1001/jamainternmed.2020.3596.

27. National Public Health Organization: Further covid 19 data from Greece. Available at: https://eody.gov.gr/en/further-covid-19-data-from-greece. Accessed July 23, 2020.

\section{Figures}



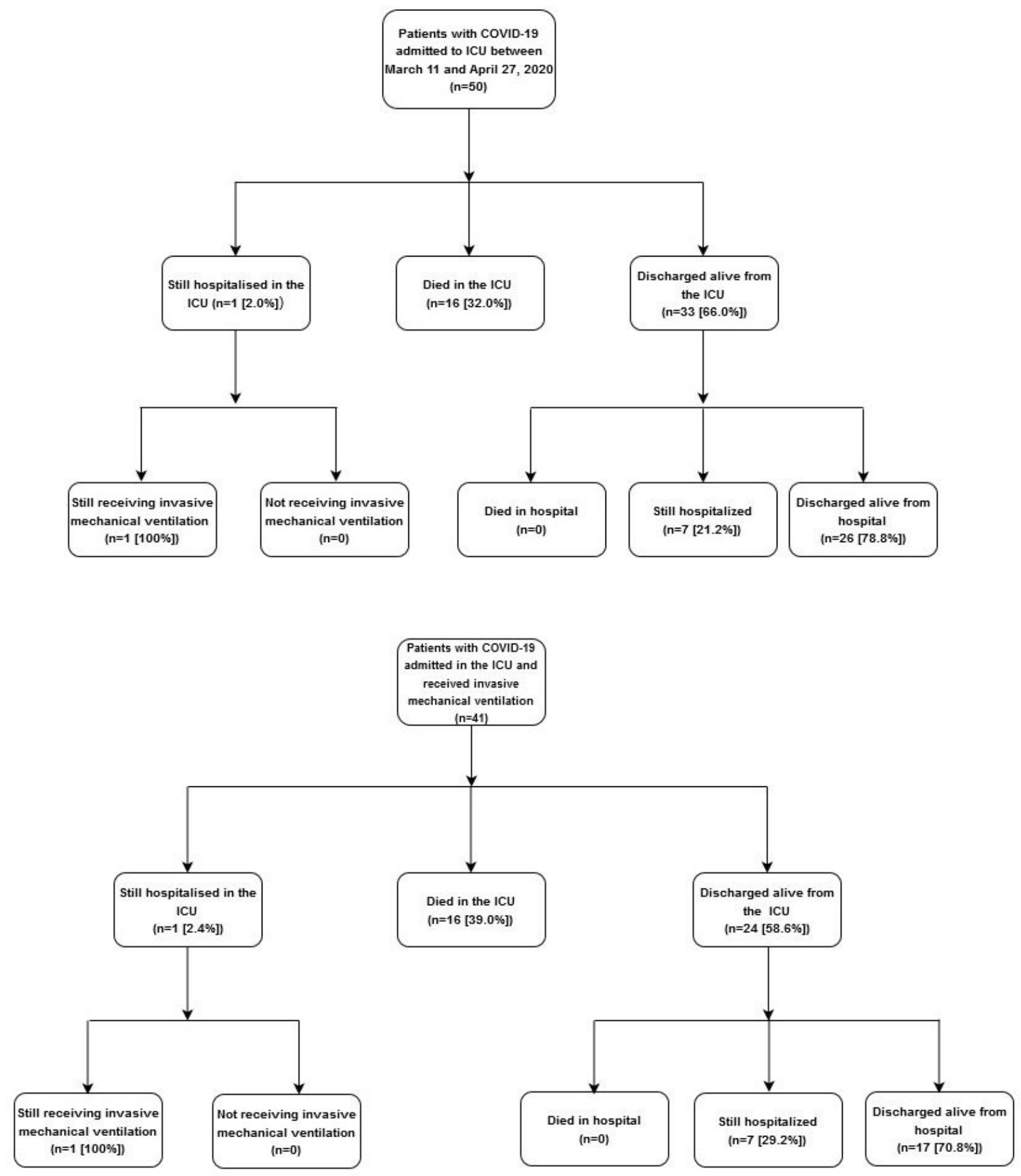

\section{Figure 1}

Flow diagram for study patients who were admitted to coronavirus disease 2019-designated ICUs (A), and received invasive mechanical ventilation (B). COVID-19 = coronavirus disease 2019; ICU = intensive care unit. 


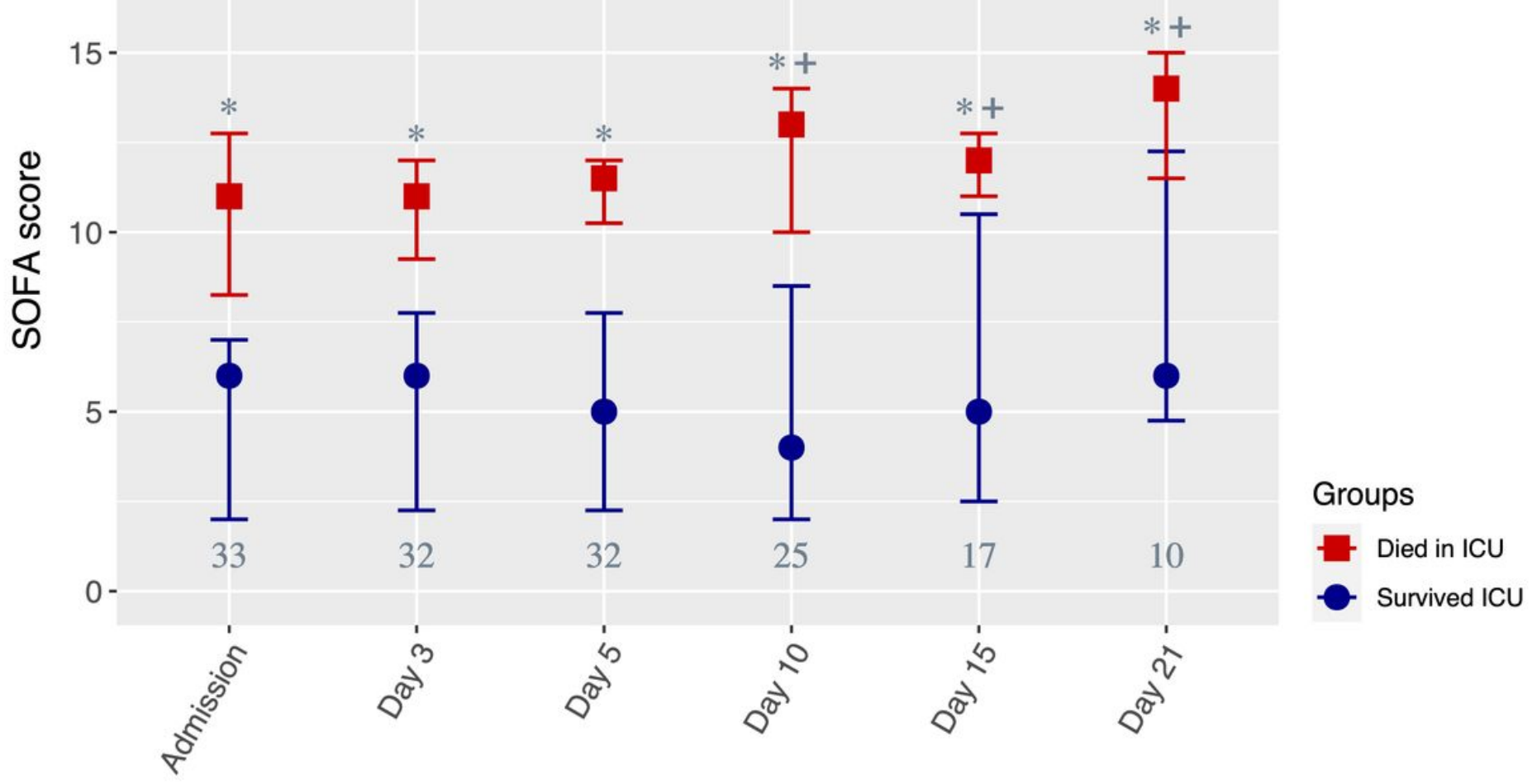

Figure 2

Serial SOFA score of patients admitted to the ICU and either survived or died in ICU. Days represent ICU days. Values are medians and error bars are interquartile ranges. Numbers represent the number $(n)$ of patients on each day. Asterisks denote significant differences between died and survived on the same ICU day ( $p<0.01$; Mann-Whitney $U$ test), whereas crosses denote significant differences in patients who died between SOFA scores on days 10, 15 and 21 and SOFA scores on admission, day 3 or day 5 ( $p<0.05$; Wilcoxon matched-pair test). SOFA = Sequential Organ Failure Assessment; ICU = intensive care unit.

\section{Supplementary Files}

This is a list of supplementary files associated with this preprint. Click to download.

- GraphicalAbstractlmage.jpg

- GraphicalAbstractlmage.jpg 